\title{
Psychoactive Drugs and Pilot Performance: A Comparison of Nicotine, Donepezil, and Alcohol Effects
}

\author{
Martin S Mumenthaler*,', Jerome A Yesavage ${ }^{1,2}$, Joy L Taylor ${ }^{1,2}$, Ruth O'Hara', Leah Friedman', Hana \\ Lee' and Helena C Kraemer' \\ 'Department of Psychiatry and Behavioral Sciences, Stanford University School of Medicine, Stanford, CA, USA; ${ }^{2}$ Department of Veterans Affairs \\ Health Care System, Palo Alto, CA, USA
}

\begin{abstract}
The cholinergic system plays a major role in cognitive abilities that are essential to piloting an aircraft: attention, learning, and memory, In previous studies, drugs that enhance the cholinergic system through different pharmacologic mechanisms have shown beneficial effects on cognition; but dissimilar cognitive measures were used and samples were not comparable. A comparison within the same cognitive tasks, within comparable samples appears desirable. Toward this aim, we compared effect sizes (ES) of performance-enhancing doses of nicotine (a nicotinic receptor agonist) and donepezil (an acetylcholinesterase inhibitor) as found in our prior work on pilot performance. We also compared cholinergic ES to those of performance-impairing doses of alcohol. In three randomized, placebo-controlled trials, we assessed the flight performance of aircraft pilots in a Frasca | 4 I simulator, testing I: the acute effects of nicotine gum 2 mg; II: the effects of administration of $5 \mathrm{mg}$ donepezil/day for 30 days; and III: the acute and $8 \mathrm{~h}$-carryover effects of alcohol after a target peak BAC of $0.10 \%$. We calculated the ES of nicotine, donepezil, and alcohol on a flight summary score and on four flight component scores. Compared to placebo, nicotine and donepezil significantly improved, while alcohol significantly impaired overall flight performance: ES (nicotine) =0.80; ES $($ donepezil) $=1.02$; ES (alcohol acute $)=-3.66$; ES (alcohol $8 \mathrm{~h})=-0.82$. Both cholinergic drugs showed the largest effects on flight tasks requiring sustained visual attention. Although the two tested cholinergic drugs have different pharmacologic mechanisms, their effects on flight performance were similar in kind and size. The beneficial effects of the cholinergic drugs on overall flight performance were large and the absolute (ie nondirectional) sizes were about one-fourth of the absolute ES of acute alcohol intoxication and roughly the same as the absolute 8 h-carryover ES of alcohol.
\end{abstract}

Neuropsychopharmacology (2003) 28, 1366-1373, advance online publication, I4 May 2003; doi:I0.1038/sj.npp. 1300202

Keywords: cholinergic agents; ethanol; cognition; psychomotor performance; psychopharmacology; aerospace medicine

\section{INTRODUCTION}

The cholinergic system of the central nervous system (CNS) has been known to play an essential role in attention, learning, and memory. Acetylcholine, the major endogenous cholinergic neurotransmitter, modulates neuronal activity through agonist effects on muscarinic and nicotinic receptors. Reduced expression of nicotinic acetylcholine receptors has been associated with reduced performance in attention, learning and memory tasks in animals and normal humans and appears to be one of the markers of the pathogenesis of Alzheimer's disease (AD) (Albuquerque et al, 2001; Gattu et al, 1997). Degeneration of cholinergic neurons in the basal forebrain and the associated loss of cholinergic neurotransmission in the cerebral cortex and

*Correspondence: MS Mumenthaler, Department of Psychiatry, School of Medicine, Stanford University, C30I, Stanford, CA 94305-5550, USA, Tel: + I 650852 3287, Fax: + I 6508523297,

E-mail: msm@stanford.edu

Received 25 October 2002; revised 06 February 2003; accepted 18 March 2003

Online publication: 27 March 2003 at http://www.acnp.org/citations/ Npp032703293/default.pdf other areas contribute significantly to the deterioration in cognitive function seen in patients with $\mathrm{AD}$ ('cholinergic hypothesis of AD') (Bartus et al, 1982; Francis et al, 1999).

In previous studies, pharmacologic enhancement of the cholinergic system has shown positive effects on cognition in animals and humans (Bartus et al, 1982; Levin and Simon, 1998; Rusted and Warburton, 1989). There are two common pharmacologic mechanisms used to increase activation of cholinergic receptors: (1) directly through administration of cholinergic agonists such as nicotine, and (2) indirectly through administration of acetylcholinesterase inhibitors (AChEIs), such as donepezil. AChEIs inhibit the breakdown of the major endogenous cholinergic agonist acetylcholine, and therefore increase the amount of acetylcholine in the synaptic cleft to bind to muscarinic and nicotinic receptors, which enhances neuronal transmission (Albuquerque et al, 2001).

\section{Cholinergic Agonists}

Rodent and primate studies have shown that nicotinic agonists play an important role in cognitive functions that involve attention, learning and memory, and such agonists 
have improved performance across memory tasks (Levin and Simon, 1998). In other animal studies, nicotine administration resulted in significant improvements in response speed and in spatial memory accuracy in rats performing the eight-arm radial maze under the influence of a $\alpha 4 \beta 2$ nicotinic receptor antagonist (Arthur and Levin, 2002). Other studies showed that chronic nicotine administration improved the working memory (WM) performance of young normal adult rats, and acute nicotine administration improved the WM performance of aged rats in the radial-arm maze (Levin et al, 1997; Levin and Torry, 1996). These findings suggest that nicotinic treatment may be effective in reducing age-associated memory impairments.

In human studies, nicotine improved discriminative sensitivity and reaction times on a computerized test of attention and information processing in $\mathrm{AD}$ patients (Sahakian et al, 1989). Other studies showed that nicotine improved attention and memory in patients with $\mathrm{AD}$ or Parkinson's disease (Min et al, 2001; Vidal, 1996). In studies with healthy volunteers, cholinergic enhancers (for example, arecoline, a muscarinic agonist, and choline, a precursor of acetylcholine) have been tested for effects on memory performance after administration of the cholinergic antagonist methscopolamine. Both drugs reversed scopolamine-induced impairment of serial learning (Sitaram et al, 1978). Within a healthy, nonsmoking sample, nicotine has improved short-term verbal memory functions (Min et al, 2001).

Two effects of nicotine, improved information processing and enhanced sensorimotor performance (Sherwood et al, 1992), are particularly relevant to driving a car or flying an airplane. Piloting an aircraft demands a high level of psychomotor coordination and cognitive skills such as sustained attention and three-dimensional thinking. Flight simulator tests are therefore well suited to study the effects of cholinergic drugs on cognition. Nicotine was the first cholinergic drug we tested on aviators in our laboratory. In our placebo-controlled study of pilots who were nonsmokers, nicotine improved overall flight simulator performance, mainly by increasing performance on approach to landing, a task that requires sustained visual attention because aviators need to monitor cockpit instrument readings over a time period of several minutes as they control their descent to the runway (Mumenthaler et al, 1998).

\section{Acetylcholinesterase Inhibitors}

A number of studies of AChEIs in both young and old humans and animals suggest a broad range of effects on attentional processes and memory. Early studies of the cholinesterase inhibitor physostigmine observed significant drug-induced improvement in humans in long-term memory of words (Davis et al, 1978), in picture recognition (Christie et al, 1981), and significant dose-dependant improvement in aged monkeys in recent visual memory (Bartus and Uehara, 1979). More recently, physostigmine administration has changed the level of arousal and selective attention to visual tasks in pattern-flash stimulus tests in human PET studies of elderly subjects (Mentis et al, 2001). In a cerebral blood flow study, with healthy human volunteers (age range 22-68 years), physostigmine was associated with improved WM efficiency as indicated by faster reaction times in a WM task for faces, and by reduced activation of cortical regions associated with WM (Furey et al, 1997). Another recent study of the cholinesterase inhibitor tetrahydroaminoacridine (THA) in young and aged healthy monkeys showed enhanced visual divided attention performance, measured via a computer task requiring simultaneous tracking of two visual targets (O’Neill et al, 1999). Cholinergic drugs have also been associated with improvements on other measures of visual attention (for example, improvement in accuracy and speed on responding on the Rapid Visual Information Processing task, a test of sustained attention). This led some reviewers to suggest that part of the benefit of cholinergic drugs upon memory performance may be mediated through the attentional components involved in WM (Everitt and Robbins, 1997; Francis et al, 1999; Muir, 1997).

In phases II and III controlled trials with $\mathrm{AD}$ patients, the cholinesterase inhibitor donepezil significantly improved cognitive function, as measured with the $\mathrm{AD}$ Assessment Scale-Cognitive subscale and the Mini-Mental State Examination, MMSE, and global clinical assessment of change compared with placebo (Rogers, 1998; Rogers et al, 1998; Rogers and Friedhoff, 1996). A study of donepezil by Ogura et al (2000) achieved effects on water maze performance similar to the nicotine effects described in the studies by Levin et al: donepezil significantly decreased errors in the radial maze in rats with central cholinergic deficits. This study suggests that donepezil can minimize learning impairments. Donepezil was the second cholinergic drug we tested on aviators in our laboratory.

In contrast to our nicotine study, we chose to administer donepezil during a 30-day period rather than as a single dose because (1) prior studies have shown that chronic donepezil administration improves cognition of AD patients (Rogers, 1998; Rogers et al, 1998); (2) there is a lack of studies on the effects of chronic donepezil administration on cognition of healthy normal subjects; and (3) a prior study found that acute donepezil administration had no significant pharmacodynamic effects on cognition (Nathan et al, 2001). In our placebo-controlled study of donepezil, the drug group performed better on a set of complex flight simulator tasks than the placebo group after a 30-day treatment (Yesavage et al, 2002).

In sum, drugs that enhance the cholinergic system, such as the cholinergic agonist nicotine and the AChEIs donepezil, have shown beneficial effects on cognitive performance even though they operate through different pharmacologic mechanisms. Both the direct cholinomimetic nicotine and the indirect cholinomimetic donepezil have been shown to improve attention, learning and memory in normals as well as in individuals with cholinergic deficits. However, previous studies have tested the cognitive effects of either a nicotinic agonist or an AChEI, but never both together on the same tasks. The goal of the present study is to compare the effects of a nicotinic agonist with an AChEI on equivalent samples performing the same cognitive tasks. We evaluated the results of two of our studies that tested comparable subjects (aviators) on the same cognitive tasks (flight-performance tasks) and compared the effect sizes (ESs) of nicotine and of donepezil. For further comparison 
of the effects of psychoactive drugs and to put the ES into perspective, we contrasted the ES of the two cholinergic performance enhancers to the ES of alcohol, a third psychoactive drug we have also tested in the flight simulator, which impaired performance of the same flight measures. Sizes of effects are better suited to estimate the practical and clinical significance of a study result than 'statistical significance' which is based on $p$ levels (Kraemer, 1992). ES is a more suitable measure particularly for a comparison of results from different studies with differing numbers of subjects, such as the present comparison, because it is independent of subject size. In contrast, 'statistical significance' can be reached at any desired significance level ( $p$ level) for any size of drug effect that is not zero, simply by increasing the sample size adequately (Cohen, 1977; Kraemer and Thieman, 1987). Thus, ES may be more helpful for clinical decision making than 'statistical significance'.

\section{METHODS}

In three separately conducted, randomized, placebo-controlled trials, we tested the effects of psychoactive drugs on the flight performance of licensed aircraft pilots in a Frasca 141 flight simulator. All studies were approved by the Human Subject Committee of Stanford University and have been carried out in accordance with the ethical standards laid down in the 1964 Declaration of Helsinki. All subjects gave written informed consent to participate and could withdraw at any time.

Below we summarize the designs of the studies (more detailed descriptions can be found in the original publications). All three studies employed the same equipment, used the same flight tasks, and applied the same flight scoring methods. We used a Frasca 141 flight simulator (Urbana, IL) linked to a specialized computer (Silicon Graphics, Mountain View, CA) that generated realistic 'through-thewindow' graphics of the environment in which the pilots flew, and collected data concerning the aircraft's flight conditions. The instrumentation and flight characteristics simulated a small single-engine aircraft similar to the common Cessna 172. The pilots received air traffic control (ATC) messages in accordance with Federal Aviation Authority (FAA) standards (FAA Order 7110.650) through a cockpit speaker system. Each ATC script contained a takeoff clearance, 16 critical en route messages, followed by instructions for approach to landing and landing. Each flight lasted $75 \mathrm{~min}$. After receiving take-off clearance, pilots were given a new ATC command every 3 min with a new heading, altitude, radio frequency, and on $50 \%$ of the legs, a new transponder (identification) code that they had to remember and dial into the cockpit panel. To increase the pilots' workload, we confronted them with three different, randomly occurring, emergency situations (carburetor icing, drop of engine oil pressure, or suddenly approaching air traffic) that demanded quick, appropriate reactions.

The scoring system of the flight simulator-computer unit produced 23 flight-performance variables (Mumenthaler et $a l, 2001 \mathrm{a}, \mathrm{b})$. The values of these variables were scores derived from errors or deviations from ideal or assigned positions or values (eg altitude in feet, heading in degrees, airspeed in knots), or reaction time in seconds. As these individual variables had different units of measurement, it was necessary to standardize the scores for each variable to a common scale such as $z$-scores. We used the sample mean and SD for each individual variable at the first baseline flight as the basis for the $z$-scores. The standardized variables were aggregated into four flight component scores: ATC communication, traffic avoidance, emergency scanning, and approach to landing. Finally, a flight summary score was computed for each flight as the mean of the $z$ standardized flight component scores. We also measured the pilots' take-off and landing performance in our studies, but did not include these components in the present analyses because prior research in our laboratory (Taylor et al, 1994, 1996) has shown low reliability and little drug effects on take-off and landing scores.

\section{Study I - Nicotine Study (Mumenthaler et al, 1998)}

This randomized, placebo-controlled, double-blind, crossover study tested the acute effects of nicotine on flight simulator performance in 16 pilots who were nonsmokers with no history of regular smoking. After being trained in the flight simulator, subjects were tested twice a day on each of two counterbalanced test days: placebo day and nicotine day. Each test day lasted $8 \mathrm{~h}$ starting with a pretreatment 'warm-up' practice flight at $16.00 \mathrm{~h}$. After the practice flight, subjects performed two test flights, the first starting at $19.30 \mathrm{~h}$, the second at $22.00 \mathrm{~h}$. They received a single dose of either nicotine polacrilex $2 \mathrm{mg}$ (SmithKline Beecham Consumer Health Care, Pittsburgh, PA) or placebo gum (confectionery gum of the same size) $30 \mathrm{~min}$ before each test flight. To disguise the presence of nicotine, one drop of hot chili (Tabasco) sauce was added to all pieces of gum. Subjects were told they would receive either nicotine or placebo and were instructed to chew the gum until a peppery taste or tingling of the gum occurs, then park the gum in their cheek until the tingling subsides, and to continue slow and intermittent chewing and parking of the gum for $20 \mathrm{~min}$. We did not observe debilitating side effects of nicotine during the test days. During the practice sessions, two subjects did not tolerate the drug (vomiting) and withdrew from the study. We compared the posttreatment flight performance (mean of the two test flights) between the nicotine and the placebo test day of each subject.

\section{Study II - Donepezil Study (Yesavage et al, 2002)}

This randomized, placebo-controlled, double-blind, parallel group study tested the effects of donepezil on flight simulator performance in 18 pilots. After being trained in the flight simulator, subjects were tested twice a day on each of 2 test days that were 30 days apart: baseline day and posttreatment day. Each test day lasted $6 \mathrm{~h}$ with the first test flight starting at $16.45 \mathrm{~h}$ and the second at $20.30 \mathrm{~h}$. During the 30 days between the 2 test days, the nine subjects randomized into Group A ingested one $5 \mathrm{mg}$ donepezil capsule/day and the nine subjects randomized into Group B ingested one placebo capsule/day. Both groups received identical looking capsules that were manufactured and randomized at the University of California, San Francisco. 
All subjects enrolled into the clinical trial at baseline completed the study and no adverse experiences were reported. We calculated change scores as the post-treatment flight performance (mean of the two test flights) minus the pretreatment flight performance (mean of the two test flights) and compared the change scores between the donepezil and the placebo group.

\section{Study III - Alcohol Study (Yesavage et al, 1994)}

This randomized, placebo-controlled, single-blind, parallel group study tested the acute and $8 \mathrm{~h}$-caryover effects of alcohol on flight simulator performance in 27 pilots. After being trained in the flight simulator, subjects were tested three times during 1 test day: the test day lasted $12 \mathrm{~h}$ and consisted of a predrink baseline flight, an acute intoxication flight at a targeted peak BAC of $0.10 \%$, and an 8 -h carryover flight $8 \mathrm{~h}$ after ingestion of the last drink. The 14 subjects randomized into Group A ingested a total of $1.14 \mathrm{ml}$ alcohol/kg bodyweight, which was divided into four drinks administered within $90 \mathrm{~min}$ (subjects older than 50 years received $8 \%$ less alcohol due to an age-related increase of peak BAC, Mundt and Ross, 1993). The 13 subjects randomized into Group B ingested a placebo drink (soda with two drops of alcohol floated on top). We calculated change scores as the postdrink flight performance (one score for acute intoxication and one for 8-h carryover) minus predrink flight performance and compared the change scores between the alcohol and the placebo group.

Table 1 provides a comparison of the design and subjects of each study.

As we expected the effects of the cholinergic drugs on flight performance to be mediated through attentional processes, we tested the pilots during the late afternoon and evening (while their attention is likely to decrease) to reach larger drug effects. In the alcohol study, on the other hand, we were interested in the acute and 8-h carryover effects (8$\mathrm{h}$ bottle-to-throttle rule of the FAA), and tested the pilots during an acute intoxication flight at around noon and a carryover flight in the evening.

For the current analyses, we calculated the ES of nicotine (mean of the two test flights), donepezil (mean of the two test flights), and alcohol (acute intoxication flight and 8-h carryover flight) on the flight summary score and on the four flight component scores: ATC communication, traffic avoidance, emergency detection, and approach to landing.

In the crossover (matched pair) study (nicotine), ES was calculated as the difference between the means of the drug and the placebo condition, $M_{\mathrm{D}}-M_{\mathrm{P}}$, divided by the standard deviation of the paired difference scores (drug day minus placebo day) (Cohen, 1988):

$$
\mathrm{ES}=\frac{M_{\mathrm{D}}-M_{\mathrm{P}}}{s_{\text {diff }}},
$$

where $s_{\text {diff }}$ is the standard deviation of the paired difference scores.

In the parallel group studies (donepezil, alcohol), ES was calculated as the difference between the means of the drug and the placebo group, $M_{1}-M_{2}$, divided by the pooled standard deviation of both the groups (drug group and placebo group) (Cohen, 1988):

$$
\mathrm{ES}=\frac{M_{1}-M_{2}}{s_{\text {pooled }}}, \quad s_{\text {pooled }}=\sqrt{\frac{s_{1}^{2}+s_{2}^{2}}{2}}
$$

The subtraction, $M_{1}-M_{2}$, was done so that the difference was positive if it was in the direction of performance improvement and negative if in the direction of deterioration. This unit free measure is extensively used in meta-

Table I Study Designs and Demographic Variables of Subjects

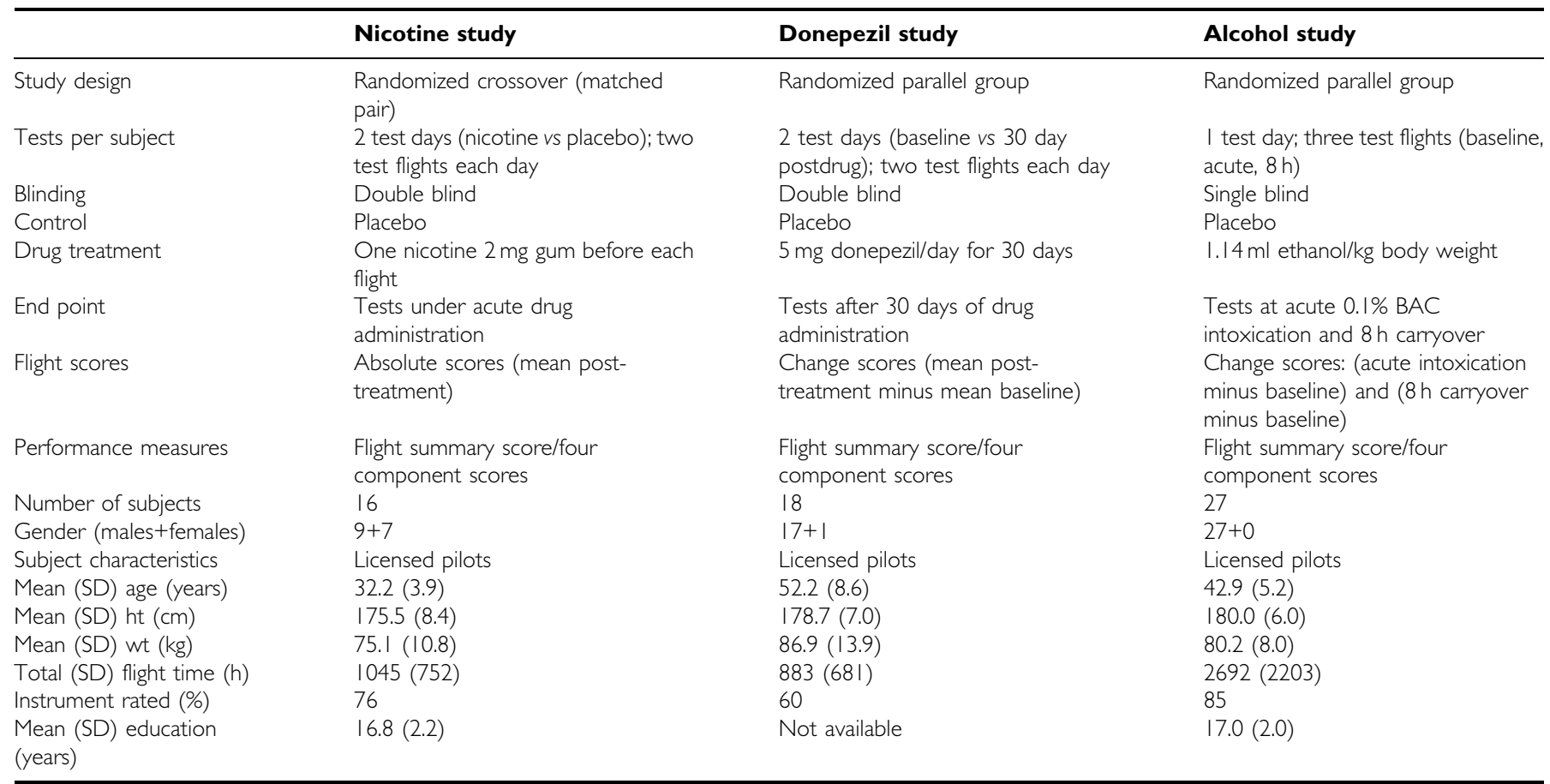


analysis and its statistical properties are well developed (Kraemer, 1992). According to the suggestions by Cohen (1988), ES around 0.2 is 'small', ES around 0.5 is 'moderate', and ES around or above 0.8 is 'large'.

\section{RESULTS}

\section{Study I-Nicotine Study}

Pilots performed significantly better during the nicotine test day (after receiving nicotine gum) than during the placebo test day (after receiving placebo gum) (see Mumenthaler et al, 1998). Table 2 shows all mean absolute flight summary scores and flight component scores of the pilots in the nicotine and the placebo condition and the $t$-values of the comparisons of the means. The ES of nicotine on overall flight performance was 'large' and mainly due to a 'moderate' effect of the drug on performance of the approach to landing (ES are presented in Table 5).

\section{Study II-Donepezil Study}

Pilots who ingested a daily dose of $5 \mathrm{mg}$ donepezil for 30 days performed significantly better on emergency detection, on approach to landing and on overall flight performance than pilots who ingested a placebo pill for 30 days (see Yesavage et al, 2002).

Table 3 shows all mean flight summary change scores and flight component change scores of the pilots in the donepezil and the placebo condition and the $t$-values of the comparison of the means. The ES of donepezil on overall flight performance was 'large' and mainly due to a 'large' effect of the drug on performance of the emergency detection and the approach to landing (Table 5).

\section{Study III - Alcohol Study}

Pilots who ingested approximately $1 \mathrm{ml}$ alcohol/kg bodyweight to reach a BAC of approximately $0.1 \%$. performed significantly worse during acute intoxication on ATC communication, on emergency detection, on approach to landing and on overall flight performance compared to pilots who ingested a placebo drink. After $8 \mathrm{~h}$ later, the overall flight performance of the pilots in the alcohol group was still significantly worse than the performance of the pilots in the placebo group (see Yesavage et al, 1994). Table 4 shows all mean flight summary change scores and flight component change scores of the pilots in the acute intoxication, $8 \mathrm{~h}$-carryover, and placebo conditions and the

Table 2 Mean (SD) Post-Treatment ${ }^{\mathrm{a}}$ Flight Summary and Component Scores of Nicotine and Placebo Group $(N=16)$

\begin{tabular}{lrrr}
\hline Flight score & Nicotine & Placebo & t-Value \\
\hline Flight summary & $0.07(0.36)$ & $-0.06(0.35)$ & $3.21 * *$ \\
ATC communication & $-0.07(0.78)$ & $0.05(0.67)$ & -1.34 \\
Traffic avoidance & $0.15(0.43)$ & $0.00(0.66)$ & 1.34 \\
Emergency detection & $-0.14(0.84)$ & $-0.26(0.81)$ & 0.64 \\
Approach to landing & $0.21(0.51)$ & $-0.05(0.51)$ & $2.41 *$ \\
*P $<0.05$ level of statistical significance. & & \\
*** $p<0.0$ I level of statistical significance. & & \\
aMean of two post-treatment flights. & &
\end{tabular}

Neuropsychopharmacology
Table 3 Mean (SD) Post-Treatment ${ }^{\mathrm{a}}$ Flight Summary and Component Change Scores of Donepezil and Placebo Group $(N=18)$

\begin{tabular}{lrlr}
\hline Flight score & Donepezil & Placebo & t-Value \\
\hline Flight summary & $0.06(0.31)$ & $-0.24(0.19)$ & $2.48^{*}$ \\
ATC communication & $-0.15(0.36)$ & $-0.08(0.50$ & -0.34 \\
Traffic avoidance & $0.11(0.60)$ & $-0.05(0.31)$ & 0.74 \\
Emergency detection & $0.33(0.76)$ & $-0.36(0.42)$ & $2.34^{*}$ \\
Approach to landing & $-0.04(0.20)$ & $-0.46(0.53)$ & $2.22 *$ \\
\hline
\end{tabular}

Positive change scores indicate performance improvement compared to baseline.

${ }^{*} p<0.05$ level of statistical significance.

a Mean of two post-treatment flights.

$t$-values of the comparisons of the means. The ES of acute alcohol intoxication on overall flight performance was 'large' and mainly due to 'large' effects of the drug on performance of the ATC communication, emergency detection, and approach to landing. At $8 \mathrm{~h}$ after drinking, the alcohol carryover effect on overall flight performance was still 'large' and mainly due to a 'moderate' effect of the drug on the emergency detection (Table 5).

\section{DISCUSSION}

All the tested drugs, nicotine, donepezil and alcohol, had 'large' effects on overall flight performance: the cholinergic drugs improved, while alcohol (acutely and $8 \mathrm{~h}$ after drinking) impaired overall flight performance compared to placebo. As could be expected, the largest drug effect was the negative impact of alcohol during acute intoxication $(\mathrm{ES}=-3.66)$. It is surprising, however, that $8 \mathrm{~h}$ after the last drink, alcohol still had a 'large' negative effect $(E S=-0.82)$ on overall flight performance. The negative effect of alcohol at that time was of roughly the same magnitude as the 'large' positive effect of the two cholinergic drugs nicotine $(E S=0.80)$ and donepezil $(E S=1.02)$. Another way of expressing this finding is that the beneficial effects of the two tested cholinergic drugs were approximately one-fourth of the detrimental effect of acute alcohol intoxication at a BAC near $0.1 \%$, the legal limit for driving a car in many countries.

To put these ESs in perspective, we cite a paper by Kazdin and Bass (1989), who demonstrated that in psychotherapy research, an ES of about 0.2 is typically found between a new treatment and an alternative standard treatment, one of about 0.5 between a new treatment and an active control treatment, and one of about 0.8 between a new treatment and a placebo treatment.

Another helpful way of demonstrating the meaning of the present ES is by calculating the area under the curve (AUC) (Figure 1). The AUC represents the standard normal cumulative distribution, where the distribution has a mean of 0 and a standard deviation of 1 . An ES of 0.8 (nicotine) represents an AUC of $71 \%$, which means that, in our study, $71 \%$ of the pilots would do better under the influence of nicotine treatment compared to placebo. In other words: a pilot has a $71 \%$ chance of performing better after taking nicotine as administered in our study. Acute alcohol 
Table 4 Mean (SD) Acute Intoxication and 8-h Carryover Flight Summary and Component Change Scores of Alcohol and Placebo Group $(N=27)$

\begin{tabular}{|c|c|c|c|c|c|c|}
\hline Flight score & Alcohol acute & Placebo acute & $t$-Value acute & Alcohol 8-h & Placebo 8-h & t-Value 8-h \\
\hline ATC communication & $-0.67(0.84)$ & $0.10(0.88)$ & $-2.57 *$ & $0.25(0.67)$ & $0.23(1.09)$ & 0.08 \\
\hline Traffic avoidance & $0.11(0.55)$ & $0.20(0.61)$ & -0.44 & $0.12(0.50)$ & $0.26(0.56)$ & -0.77 \\
\hline Approach to landing & $-0.57(0.7 \mathrm{I})$ & $0.30(1.12)$ & $-2.55 *$ & $0.30(0.75)$ & $0.32(0.99)$ & -0.08 \\
\hline
\end{tabular}

Negative change scores indicate performance impairment compared to baseline.

$* p<0.05$ level of statistical significance.

** $p<0.0$ l level of statistical significance.

**** $p<0.00$ I level of statistical significance.

Table 5 ES of Nicotine, Donepezil, and Alcohol on Flight Summary and Component Scores

\begin{tabular}{lcclc}
\hline Flight score & $\begin{array}{c}\text { Nicotine } \\
\text { ES }\end{array}$ & $\begin{array}{c}\text { Donepezil } \\
\text { ES }\end{array}$ & $\begin{array}{c}\text { Alcohol acute } \\
\text { intoxication } \\
\text { ES }\end{array}$ & $\begin{array}{c}\text { Alcohol } 8 \mathbf{~ h} \\
\text { carryover } \\
\text { ES }\end{array}$ \\
\hline Flight summary & $0.80 * *$ & $1.02 *$ & $-3.66 * * *$ & $-0.82 *$ \\
ATC communication & -0.34 & -0.16 & $-0.99 *$ & 0.03 \\
Traffic avoidance & 0.34 & 0.35 & -0.17 & -0.30 \\
Emergency detection & 0.16 & $0.99 *$ & $-1.51 * * *$ & -0.75 \\
Approach to landing & $0.60 *$ & $0.94 *$ & $-0.98 *$ & -0.03 \\
\hline
\end{tabular}

Positive ES means that the drug improved performance compared to placebo; negative ES means that the drug impaired performance compared to placebo. ${ }^{*} p<0.05$ level of statistical significance.

$* * 0<0.0$ I level of statistical significance.

**** $p<0.00$ I level of statistical significance.

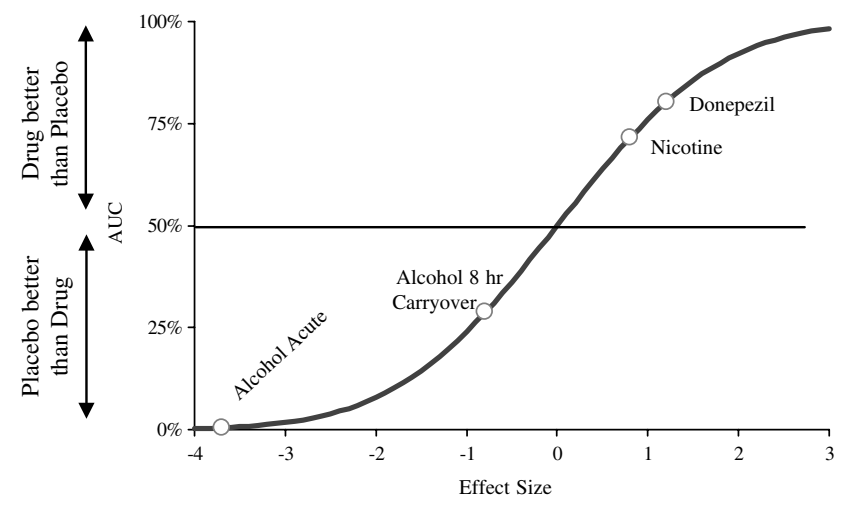

Figure I Interpretation of ES (standardized mean differences) in terms of $A \cup C$, which is the probability that drug response is greater than placebo response.

intoxication of approximately $0.10 \%$ BAC led to an ES of -3.6 , which means that $99.6 \%$ of the pilots would show flight-performance impairment under the tested conditions. At $8 \mathrm{~h}$ after the last alcoholic drink, the chance of still being impaired in flying an airplane is $71 \%(\mathrm{ES}=-0.77)$.

A previous study from our laboratory explains how our flight simulator measures may translate to measures used in other studies of cholinergic effects on cognitive performance (Taylor et al, 2000). In that study, speed and WM measures showed the strongest correlation with our flight summary score, accounting for $33 \%$ of the variance of the flight summary score. Additional significant predictors of our flight summary score were visual associative memory, tracking, and motor coordination measures. There was also evidence for specific ability relations: (1) between the pilot's performance on ATC communication and backward digit span (after partialling out speed of processing); (2) between approach to landing and psychomotor tracking performance (dual task tracking alone and dual error). Performance on air traffic avoidance was related to a cognitive measure of overall speed of processing.

We note that the only flight component score that was at least 'moderately' affected by all three tested drugs $(|\mathrm{ES}|>0.60)$ was the score of the approach to landing task. This flight task appears to demand the highest amount of sustained visual attention, which might explain its high sensitivity to the effects of the tested psychoactive drugs that have previously been found to affect attentional processes. Comparison of the ES on the flight summary score and on the flight component scores between the two cholinergic drugs shows interesting similarities. The two different mechanisms of cholinergic enhancement (direct agonist nicotine and AChEI donepezil) showed beneficial effects that were similar in magnitude on overall flight performance as well as on traffic avoidance, and on approach to landing. The fact that the two cholinergic drugs had a 'moderate' to 'large' positive effect on the flight tasks, which require a particularly high amount of sustained visual attention (approach to landing), indicates the similarity of the pharmacodynamics of the two cholinergic drugs in regard to human performance enhancement.

We did not expect the overall effect of donepezil to be larger than that of nicotine in our studies. Possible explanations for this finding include (1) the chronic 30day (donepezil) $v s$ the acute single-dose (nicotine) drug administration; (2) the difference in the subjects' mean age between the two studies; and (3) the difference in the subjects' flight experience and training. The mean age of the subjects in the donepezil group was 20 years higher than that in the nicotine group and one might speculate that the performance of older pilots benefits more from a cholinergic drug than that of younger pilots. The pilots of the donepezil study, on average, had less flight experience (mean total flight time $=08.83 \mathrm{~h}$ ) than those of the nicotine study (mean $=10.45 \mathrm{~h}$ ) and only $60 \%$ of the pilots of the donepezil study were trained to fly under instrument flight rules (IFR), compared to $76 \%$ in the nicotine study. One might speculate that less-experienced and less-trained pilots 
benefit more from cholinergic drugs. While the present study was not designed to test such hypotheses, future studies might be designed to investigate how drug administration, age, and the amount of flight experience and training mediate cholinergic drug effects on flight performance.

To allow evaluation of the practical significance of our study's findings, we note some of its strengths and limitations. The strengths that make this comparison of two cholinergic drugs and alcohol unique are that it is based on three clinical trials that: (1) were conducted at the same laboratory (same 'site'); (2) used subjects with similar demographics; (3) investigated subjects who had a key characteristic in common: they were all licensed pilots; and (4) applied identical outcome measures (flight performance in the same simulator). The limitations of the comparison study are that it is based on three clinical trials with partly different designs (parallel group $v s$ crossover; different means of subject age). Generally, a crossover design is more valid and powerful to test the effects of a drug on flight performance, if there is no evidence that either the drug or the testing procedure have any carryover effects on subsequent performance. Under these conditions, a crossover design reduces the influence of the between-subjects variability and therefore results in higher statistical power. This is the preferred design when acute drug effects on performance (such as acute nicotine effects) are investigated. However, when a drug has little acute effects on performance because it requires a longer administration period (typically drugs with longer elimination half lives), a parallel group design is preferred, as carryover effects would require long washout periods during the study. The elimination half-life of donepezil is about $70 \mathrm{~h}$; that of nicotine is about $2 \mathrm{~h}$. Our expectation was that administration of two $2 \mathrm{mg}$ nicotine gum in 1 day to nonsmokers would not have any pharmacologic carryover effects, but that a 30-day administration of $5 \mathrm{mg}$ donepezil/day would have significant pharmacologic carryover effects. This is why we designed the nicotine trial as a crossover study and the donepezil trial as a parallel group study. To shed more light on the similarities and differences of effects of cholinergic drugs with different pharmacologic mechanisms, future investigations might conduct head-to-head comparisons of direct and indirect cholinergic drugs in one study applying a parallel group design comparing subject groups with a similar mean age.

\section{ACKNOWLEDGEMENTS}

This research was supported in part by NIMH Grant 40041; NIA Grant AG17824; the Sierra-Pacific Mental Illness Research, Education, and Clinical Center (MIRECC); the Alcohol Beverage Medical Research Foundation; the Swiss Foundation for Alcohol Research; the Swiss National Science Foundation; and the Medical Research Service of the Department of Veterans Affairs.

\section{REFERENCES}

Albuquerque EX, Santos MD, Alkondon M, Pereira EFR, Maelicke A (2001). Modulation of nicotinic receptor activity in the central nervous system: a novel approach to the treatment of Alzheimer disease. Alzheimer Dis Assoc Disord 15(Suppl 1): S19-S25.

Arthur D, Levin ED (2002). Chronic inhibition of alpha4beta2 nicotinic receptors in the ventral hippocampus of rats: impacts on memory and nicotine response. Psychopharmacology (Berl) 160: $140-145$.

Bartus RT, Dean RLD, Beer B, Lippa AS (1982). The cholinergic hypothesis of geriatric memory dysfunction. Science 217: 408414.

Bartus RT, Uehara Y (1979). Physostigmine and recent memory: effects in young and aged nonhuman primates. Science 206: 1087-1089.

Christie JE, Shering A, Ferguson J, Glen AI (1981). Physostigmine and arecoline: effects of intravenous infusions in Alzheimer presenile dementia. BrJ Psychiatry 138: 46-50.

Cohen J (1977). Statistical Power Analysis for the Behavioral Sciences, rev edn Academic Press: New York.

Cohen J (1988). Statistical Power Analysis for the Behavioral Sciences, 2nd edn, rev edn Lawrence Earlbaum Associates: Hillsdale, NJ.

Davis KL, Mohs RC, Tinklenberg JR, Pfefferbaum A, Hollister LE, Kopell BS (1978). Physostigmine: improvement of long-term memory processes in normal humans. Science 201: 272-274.

Everitt BJ, Robbins TW (1997). Central cholinergic systems and cognition. Ann Rev Psychol 48: 649-684.

Francis PT, Palmer AM, Snape M, Wilcock GK (1999). The cholinergic hypothesis of Alzheimer's disease: a review of progress. 66: 137-147.

Furey ML, Pietrini P, Haxby JV, Alexander GE, Lee HC, VanMeter J et al (1997). Cholinergic stimulation alters performance and task-specific regional cerebral blood flow during working memory. Proc Natl Acad Sci USA 94: 6512-6516.

Gattu M, Pauly JR, Boss KL, Summers JB, Buccafusco JJ (1997). Cognitive impairment in spontaneously hypertensive rats: role of central nicotinic receptors. I. Brain Res 771: 89-103.

Kazdin AE, Bass D (1989). Power to detect differences between alternative treatments in comparative psychotherapy outcome research. J Consult Clin Psychol 57: 138-147.

Kraemer HC (1992). Reporting the size of effects in research studies to facilitate assessment of practical or clinical significance. Psychoneuroendocrinology 17: 527-536.

Kraemer HC, Thieman S (1987). How many Subjects? Sage Publications: Newbury Park.

Levin ED, Kaplan S, Boardman A (1997). Acute nicotine interactions with nicotinic and muscarinic antagonists: working and reference memory effects in the 16-arm radial maze. Behav Pharmacol 8: 236-242.

Levin ED, Simon BB (1998). Nicotinic acetylcholine involvement in cognitive function in animals. Psychopharmacology 138: 217-230.

Levin ED, Torry D (1996). Acute and chronic nicotine effects on working memory in aged rats. Psychopharmacology (Berl) 123: 88-97.

Mentis MJ, Sunderland T, Lai J, Connolly C, Krasuski J, Levine B et al (2001). Muscarinic versus nicotinic modulation of a visual task. A pet study using drug probes. Neuropsychopharmacology 25: 555-564.

Min SK, Moon IW, Ko RW, Shin HS (2001). Effects of transdermal nicotine on attention and memory in healthy elderly nonsmokers. Psychopharmacology (Berl) 159: 83-88.

Muir JL (1997). Acetylcholine, aging, and Alzheimer's disease. Pharmacol, Biochem Behav 56: 687-696.

Mumenthaler MS, O'Hara R, Taylor JL, Friedman L, Yesavage JA (2001a). Influence of the menstrual cycle on flight simulator performance after alcohol ingestion. J Stud Alcohol 62: 422-433.

Mumenthaler MS, O’Hara R, Taylor JL, Yesavage JA (2001b). Relationship between variation in estradiol and progesterone levels across the menstrual cycle and human performance. Psychopharmacology 155: 198-203. 
Mumenthaler MS, Taylor JL, O'Hara R, Yesavage JA (1998). Influence of nicotine on simulator flight performance in nonsmokers. Psychopharmacology 140: 38-41.

Mundt JC, Ross LE (1993). Methodological issues for evaluation of alcohol and other drug effects: examples from flight-simulator performance. Behav Res Methods Inst Comput 253: 360-365.

Nathan PJ, Baker A, Carr E, Earle J, Jones M, Nieciecki M et al (2001). Cholinergic modulation of cognitive function in healthy subjects: acute effects of donepezil, a cholinesterase inhibitor. Hum Psychopharmacol 16: 481-483.

Ogura H, Kosasa T, Kuriya Y, Yamanishi Y (2000). Donepezil, a centrally acting acetylcholinesterase inhibitor, alleviates learning deficits in hypocholinergic models in rats. Methods Fundam Exp Clin Pharmacol 22: 89-95.

O’Neill J, Fitten LJ, Siembieda DW, Crawford KC, Halgren E, Fisher A et al (1999). Divided attention-enhancing effects of AF102B and THA in aging monkeys. Psychopharmacology 143: 123-130.

Rogers SL (1998). Perspectives in the management of Alzheimer's disease: clinical profile of donepezil. Dementia Geriatric Cogn Disord 9(Suppl 3): 29-42.

Rogers SL, Doody RS, Mohs RC, Friedhoff LT (1998). Donepezil improves cognition and global function in Alzheimer disease: a 15-week, double-blind, placebo-controlled study. Donepezil Study Group. Arch Intern Med 158: 1021-1031.

Rogers SL, Friedhoff LT (1996). The efficacy and safety of donepezil in patients with Alzheimer's disease: results of a US multicentre, randomized, double-blind, placebo-controlled trial. The Donepezil Study Group. Dementia 7: 293-303.

Rusted JM, Warburton DM (1989). Cognitive models and cholinergic drugs. Neuropsychobiology 21: 31-36.
Sahakian B, Jones G, Levy R, Gray J, Warburton D (1989). The effects of nicotine on attention, information processing, and short-term memory in patients with dementia of the Alzheimer type. Br J Psychiatry 154: 797-800.

Sherwood N, Kerr JS, Hindmarch I (1992). Psychomotor performance in smokers following single and repeated doses of nicotine gum. Psychopharmacology 108: 432-436.

Sitaram N, Weingartner H, Gillin JC (1978). Human serial learning: enhancement with arecholine and choline impairment with scopolamine. Science 201: 274-276.

Taylor JL, O'Hara R, Mumenthaler MS, Yesavage JA (2000). Relationship of CogScreen-AE to flight simulator performance and pilot age. Aviat Space Environ Med 71: 373-380.

Taylor JL, Dolhert N, Friedman L, Mumenthaler MS, Yesavage JA (1996). Alcohol elimination and simulator performance of male and female aviators: a preliminary report. Aviat Space Environ Med 67: 407-413.

Taylor JL, Dolhert N, Morrow D, Friedman L, Yesavage JA (1994). Acute and 8-hour effects of alcohol $(0.08 \%$ BAC) on younger and older pilots' simulator performance. Aviat Space Environ Med 65: 718-725.

Vidal C (1996). Nicotinic receptors in the brain. Molecular biology, function, and therapeutics. Mol Chem Neuropathol 28: 3-11.

Yesavage JA, Dolhert N, Taylor JL (1994). Flight simulator performance of younger and older aircraft pilots: effects of age and alcohol. J Am Geriatr Soc 42: 577-582.

Yesavage JA, Mumenthaler MS, Taylor JL, Friedman L, O'Hara R, Sheikh J et al (2002). Donepezil and flight simulator performance: effects on retention of complex skills. Neurology 59: 123125. 